\title{
Article \\ Contribution of Ascorbate and Glutathione in Endobacteria Bacillus subtilis-Mediated Drought Tolerance in Two Triticum aestivum L. Genotypes Contrasting in Drought Sensitivity
}

\author{
Dilara Maslennikova and Oksana Lastochkina *(D)
}

check for

updates

Citation: Maslennikova, D.; Lastochkina, O. Contribution of Ascorbate and Glutathione in Endobacteria Bacillus

subtilis-Mediated Drought Tolerance in Two Triticum aestivum L. Genotypes Contrasting in Drought Sensitivity. Plants 2021, 10, 2557. https:// doi.org/10.3390/plants10122557

Academic Editors: Marina Tucci and Monica De Palma

Received: 13 October 2021

Accepted: 19 November 2021

Published: 23 November 2021

Publisher's Note: MDPI stays neutral with regard to jurisdictional claims in published maps and institutional affiliations.

Copyright: (c) 2021 by the authors. Licensee MDPI, Basel, Switzerland. This article is an open access article distributed under the terms and conditions of the Creative Commons Attribution (CC BY) license (https:/ / creativecommons.org/licenses/by/ $4.0 /)$.
Institute of Biochemistry and Genetics, Subdivision of the Ufa Federal Research Centre of the Russian Academy of Sciences, 450054 Ufa, Russia; dishaoil@mail.ru

* Correspondence: oksanaibg@gmail.com

\begin{abstract}
We evaluated the effect of endobacteria Bacillus subtilis (strain 10-4) as a co-inoculant for promoting plant growth and redox metabolism in two contrasting genotypes of Triticum aestivum L. (wheat): Ekada70 (drought tolerant (DT)) and Salavat Yulaev (drought susceptible (DS)) in early stages of adaptation to drought (12\% PEG-6000). Results revealed that drought reduced growth and dramatically augmented oxidative stress markers, i.e., hydrogen peroxide $\left(\mathrm{H}_{2} \mathrm{O}_{2}\right)$ and lipid peroxidation (MDA). Furthermore, the depletion of ascorbate (AsA) and glutathione (GSH), accompanied by a significant activation of ascorbate peroxidase (APX) and glutathione reductase (GR), in both stressed wheat cultivars (which was more pronounced in DS genotype) was found. B. subtilis had a protective effect on growth and antioxidant status, wherein the stabilization of AsA and GSH levels was revealed. This was accompanied by a decrease of drought-caused APX and GR activation in DS plants, while in DT plants additional antioxidant accumulation and GR activation were observed. $\mathrm{H}_{2} \mathrm{O}_{2}$ and MDA were considerably reduced in both drought-stressed wheat genotypes because of the application of B. subtilis. Thus, the findings suggest the key roles in $B$. subtilis-mediated drought tolerance in DS cv. Salavat Yulaev and DT cv. Ekada70 played are AsA and GSH, respectively; which, in both cases, resulted in reduced cell oxidative damage and improved growth in seedlings under drought.
\end{abstract}

Keywords: endophytic bacteria; Bacillus subtilis; Triticum aestivum L.; drought; tolerance; hydrogen peroxide; malondialdehyde; ascorbate; glutathione

\section{Introduction}

Drought is one of the most dominant abiotic stresses hindering growth and yield (by up to $80 \%$ ) in crop plants and affects about $64 \%$ of the world's agricultural lands [1-4]. Drought conditions impair the general metabolism of plants at physiological, biochemical, and molecular levels, leading to growth inhibition. Drought causes an overproduction of reactive oxygen species (ROS), which leads to a change in cellular redox homeostasis, inactivation of membrane-bound proteins, decreased membrane fluidity, DNA damage, and inhibition of protein synthesis and enzymatic activity $[1,3,5,6]$. In nature, plants are equipped with various defense systems to counter external threats, including enzymatic and non-enzymatic antioxidants [5,6]. However, their protective effects are insufficient under severe drought conditions, resulting in significant yield losses in all types of crops, including such strategically important food cereals as bread wheat (Triticum aestivum L.). This creates a natural demand for new approaches that enhance natural plant defense systems. The negative effects of abiotic stresses on plants, drought in particular, can be successfully overcome by plant growth promoting bacteria (PGPB), which are natural inhabitants of the rhizosphere soil [7-10].

Endophytic PGPB, particularly Bacillus subtilis, are a cheap eco-friendly biological strategy for improving plant growth/productivity under drought conditions. They func- 
tion by activating the natural defense mechanisms of host plants without causing a negative impact on them, the environment, or human health $[7,8,11]$. The ability of $B$. subtilis to improve wheat growth and tolerance under various abiotic stresses has been reported in many studies $[7,10,12,13]$. It is presumed that Bacillus exerts a protective effect on stressed plants by colonizing external (rhizobacteria) and/or internal (endobacteria) host tissue, positively modulating plant metabolism, improving the absorption of water and nutrients by producing various bioactive compounds, and inducing multiple plant defense reactions $[10-12,14]$. However, many aspects of the interaction between endophytic B. subtilis and plants (wheat) under drought stress remain unclear and require further detailed investigation. Additionally, the effectiveness of the same B. subtilis strain can vary depending on plant genotype, environmental stresses, and many other factors [4].

A crucial step in improving drought tolerance in plants is detoxification of stresscaused ROS by utilizing an antioxidant defense mechanism that involves a number of antioxidants, both enzymatic (superoxide dismutase, peroxidase, catalase, ascorbate peroxidase (APX), and glutathione reductase (GR)) and non-enzymatic (ascorbate (AsA), glutathione (GSH), cysteine, proline, flavonoids, carotenoids, and tocopherol) [5,6,15]. There is now substantial evidence that different cereals inoculated with PGPBs can survive under abiotic stress by reducing stress-caused oxidative damages as judged by lipid peroxidation and electrolytes leakages $[7,10,14,16-18]$. It has also been demonstrated that Bacillus leads to changes in the balance of ROS and the activity of antioxidant enzymes in wheat under drought $[8,10]$, which plays an important role in the manifestation of pre-adaptive and anti-stress action of this bacterium on plants [8,10,19]. Among antioxidants, AsA and GSH are the most important in plants. Both molecules scavenge ROS and are components of the pivotal antioxidant Foyer-Halliwell-Asada pathway or AsA-GSH cycle $[5,6]$. The studies shows that wheat plant responses to drought-induced oxidative stress involve AsA-GSH cycle metabolism [6,15]. Despite the accumulated data on the ability of $B$. subtilis to reduce drought-caused oxidative damages in plants, it is still not clear how exactly they can influence the AsA-GSH metabolism, and what their contribution is to the bacterial-mediated drought tolerance of different wheat genotypes.

The aim of the present study was to assess the influences of endobacteria $B$. subtilis 10-4 on the growth, cellular oxidative status (MDA, $\mathrm{H}_{2} \mathrm{O}_{2}$ ), and the components of the AsA-GSH complex (AsA, GSH, GSSG, APX, GR) in two wheat (T. aestivum L.) genotypes with contrasting drought sensitivity and grown under drought (12\% PEG-6000) in early stages of ontogenesis.

\section{Results}

\subsection{Growth Attributes}

Analysis carried out in B. subtilis-treated wheat seeds three days post germination in the presence of 12\% PEG-6000 (drought simulation) revealed that drought significantly decreased the germination rate by 57\% (under control 97\%) and 39\% (under control 96\%) for DT cv. Ekada70 and DS cv. Salavat Yulaev, respectively (Table 1). Seed treatment with $B$. subtilis 10-4 improved the percentage of seed germination up to $74 \%$ for DT genotype, while for DS genotype an inhibitory effect (the percentage of germination was only $27 \%$ ) was observed. However, under normal growth conditions ( $0 \%$ PEG) B. subtilis 10-4 contributed to some increase in the seed germination of both wheat genotypes (Table 1).

Further analysis was carried out in early wheat seedlings hydroponically grown during four days under normal conditions and then transferred to $12 \%$ PEG-6000 (drought) for $7 \mathrm{~h}$. It was observed that exposure to drought for $7 \mathrm{~h}$ led to inhibition in the growth of early wheat seedlings in both genotypes (Table 1). Drought reduced the length of shoots and roots by $15-20 \%$ in DT cv. Ekada70 (Table 1), and by $10-25 \%$ in DS cv. Salavat Yulaev (Table 1). Pre-sowing seed treatment with B. subtilis 10-4 mitigated the negative impact of drought on the growth of early wheat seedlings of both genotypes. Under normal (non-stressed) growth conditions, B. subtilis 10-4 also demonstrated growth-promoting activity on both genotypes with better effect on DT cv. Ekada70. 
Table 1. Effect of drought (12\% PEG) on seed germination (three days after sowing) percentage and length of roots and shoots of four-days old T. aestivum L. (wheat) seedlings of drought-tolerant (DT) cv. Ekada70 and drought-susceptible (DS) cv. Salavat Yulaev, non-inoculated (C) and pre-inoculated with endobacterial B. subtilis strain 10-4 (BS). Drought exposure time $-7 \mathrm{~h}$. In the table presented is the average data of three repetitions $(n=50)$.

\begin{tabular}{|c|c|c|c|c|c|c|c|c|c|}
\hline \multirow{3}{*}{\multicolumn{2}{|c|}{ Growth Parameters }} & \multicolumn{4}{|c|}{ 0\% PEG (Non-Stressed) } & \multicolumn{4}{|c|}{ 12\% PEG (Stressed) } \\
\hline & & \multicolumn{2}{|c|}{$\begin{array}{c}\text { Control } \\
\text { (Non-Inoculated) }\end{array}$} & \multicolumn{2}{|c|}{ BS } & \multicolumn{2}{|c|}{$\begin{array}{c}\text { Control } \\
\text { (Non-Inoculated) }\end{array}$} & \multicolumn{2}{|c|}{ BS } \\
\hline & & DT & DS & DT & DS & DT & DS & DT & DS \\
\hline Seed gerr & tion $(\%)$ & $97 \pm 1$ & $96 \pm 2$ & $99 \pm 1$ & $97 \pm 1$ & $57 \pm 2$ & $39 \pm 3$ & $74 \pm 2 *$ & $42 \pm 2$ \\
\hline \multirow{2}{*}{$\begin{array}{l}\text { Lenght } \\
(\mathrm{cm})\end{array}$} & Roots & $2.40 \pm 0.12$ & $3.42 \pm 0.09$ & $2.59 \pm 0.10^{*}$ & $3.54 \pm 0.14 *$ & $2.16 \pm 0.10$ & $3.25 \pm 0.09$ & $2.41 \pm 0.09 *$ & $3.51 \pm 0.09$ * \\
\hline & Shoots & $3.05 \pm 0.05$ & $3.60 \pm 0.07$ & $3.26 \pm 0.05^{*}$ & $3.65 \pm 0.06$ & $2.44 \pm 0.05$ & $3.29 \pm 0.07$ & $3.24 \pm 0.05 *$ & $3.57 \pm 0.06^{*}$ \\
\hline \multirow{2}{*}{ FW (g) } & Roots & $0.60 \pm 0.01$ & $0.99 \pm 0.02$ & $0.65 \pm 0.03$ & $0.85 \pm 0.09$ & $0.53 \pm 0.01$ & $0.76 \pm 0.02$ & $0.56 \pm 0.01$ & $0.71 \pm 0.06$ \\
\hline & Shoots & $0.66 \pm 0.02$ & $0.94 \pm 0.05$ & $0.63 \pm 0.02$ & $1.03 \pm 0.02 *$ & $0.38 \pm 0.01$ & $0.43 \pm 0.04$ & $0.41 \pm 0.06$ & $0.43 \pm 0.008$ \\
\hline \multirow{2}{*}{ DW (g) } & Roots & $0.065 \pm 0.001$ & $0.08 \pm 0.009$ & $0.059 \pm 0.007$ & $0.07 \pm 0.01$ & $0.06 \pm 0.003$ & $0.17 \pm 0.03$ & $0.062 \pm 0.02$ & $0.16 \pm 0.008$ \\
\hline & Shoots & $0.050 \pm 0.002$ & $0.08 \pm 0.007$ & $0.057 \pm 0.003$ & $0.09 \pm 0.008$ & $0.04 \pm 0.007$ & $0.09 \pm 0.01$ & $0.05 \pm 0.006$ & $0.09 \pm 0.005$ \\
\hline
\end{tabular}

* indicates a significant difference between the means at the level of $p<0.05$.

\section{2. $\mathrm{H}_{2} \mathrm{O}_{2}$ and Lipid Peroxidation (MDA)}

It was observed that drought led to significant hydrogen peroxide $\left(\mathrm{H}_{2} \mathrm{O}_{2}\right)$ generation in root tissues of both wheat genotypes (Figure 1). In the roots of DT cv. Ekada70, $\mathrm{H}_{2} \mathrm{O}_{2}$ levels increased by about $130 \%$ with a peak at $1 \mathrm{~h}$ of drought exposure (Figure 1a). In the roots of DS cv. Salavat Yulaev, a transit (almost by 300\%) increase in $\mathrm{H}_{2} \mathrm{O}_{2}$ was recorded at $0.5 \mathrm{~h}$ of stress (Figure $1 \mathrm{~b}$ ). Pre-treatment with B. subtilis 10-4 contributed to the maintenance of $\mathrm{H}_{2} \mathrm{O}_{2}$ in both genotypes at levels similar to control values (Figure $1 \mathrm{a}, \mathrm{b}$ ). It should also be noted that in non-stressed plants the initial $\mathrm{H}_{2} \mathrm{O}_{2}$ concentration was higher in DT cv. Ekada70 in comparison with plants of DS cv. Salavat Yulaev in the same growth conditions (Figure 1a,b).

A remarkable increase (by 260\%) in lipid peroxidation (MDA) was observed after $7 \mathrm{~h}$ of drought exposure in DS cv. Salavat Yulaev (Figure 1c). In contrast, a corresponding increase of MDA in DT cv. Ekada70 seedlings was only 130\%. Conversely, MDA was significantly reduced in the stressed wheat seedlings pre-treated with B. subtilis $10-4$. This effect was more pronounced (almost reaching control levels) for seedlings of DT genotype (Figure 1c), but in seedlings of DS genotype MDA declined by $62 \%$. Additionally, the results summarized in Figure 1c show that under normal growth conditions the concentration of MDA did not change in bacterial-inoculated DT genotype, but a slight increase (by $115 \%$ ) in DS genotype in comparison with non-inoculated control wheat seedlings was observed under drought conditions.

\subsection{Ascorbic Acid (AsA) and Glutathione (GSH)}

This study demonstrated differing ASA and GSH content in young DT cv. Ekada70 vs. DS cv. Salavat Yulaev wheat seedlings (Figure 2). So, under normal growth conditions, seedlings of cv. Ekada70 were characterized by an insignificant, but reliably higher (by $124 \%$ ) GSH content (Figure 2a) compared to seedlings of cv. Salavat Yulaev (Figure 2b). At the same time, the seedlings of DS cv. Salavat Yulaev contained significantly more AsA (160\%). Pre-treatment with B. subtilis 10-4 led to additional AsA accumulation in seedlings of both cultivars, with the greatest accumulation throughout the experiment observed in DT cv. Ekada70 (approximately 150\% of control). Whereas, in the cv. Salavat Yulaev it was in the range of 123-125\% relative to the control level. In Bacillus-inoculated plants the GSH did not change significantly, although a slight accumulation was observed. Additionally, the level of oxidized GSH (GSSG) in plants of both cultivars pre-inoculated with B. subtilis 10-4 remained unchanged (Figure 2e,f). This indicates the absence of a negative effect of bacterial treatment on the physiological state of plants. 

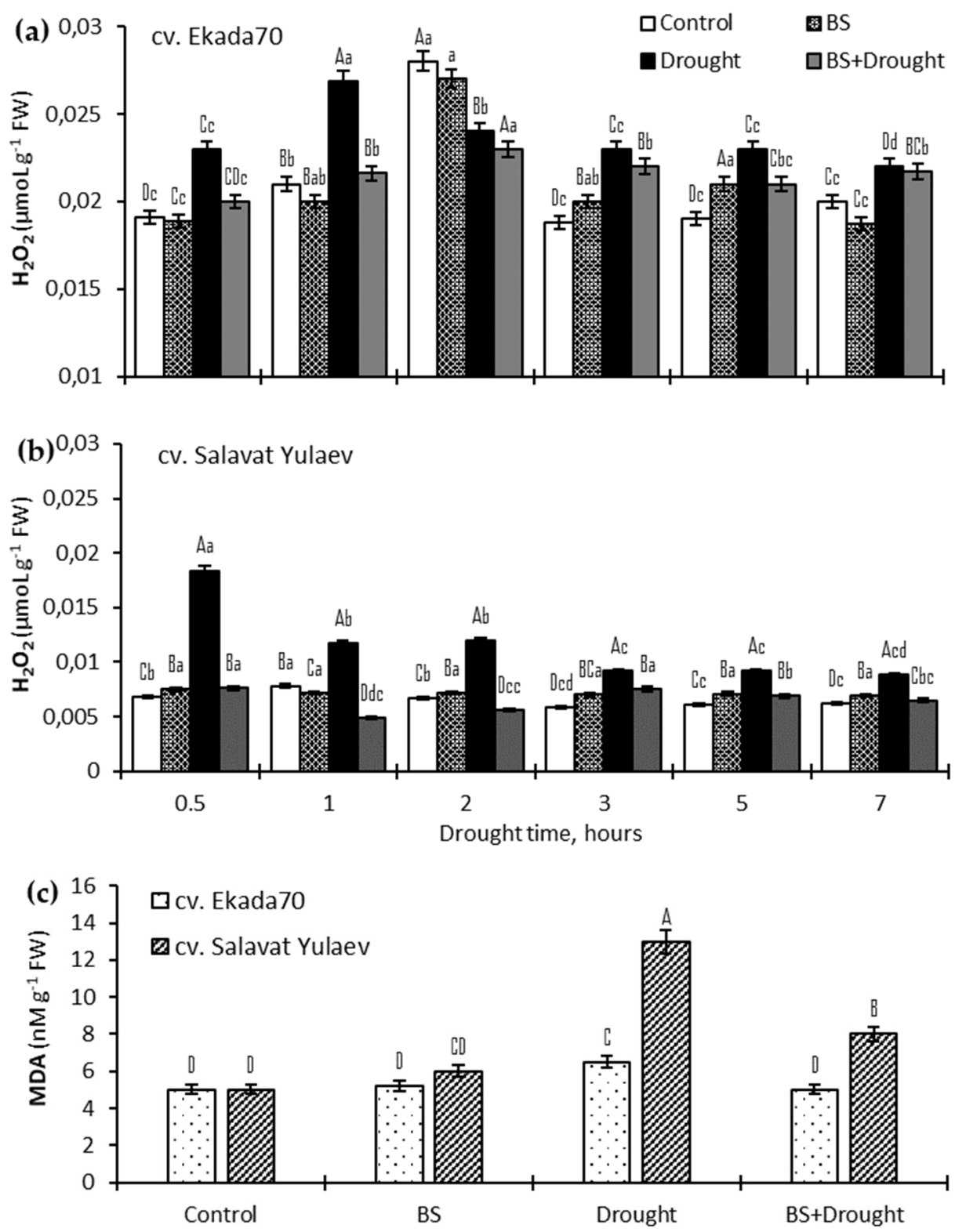

Figure 1. Influence of endobacteria B. subtilis 10-4 (BS) pre-treatment on hydrogen peroxide $\left(\mathrm{H}_{2} \mathrm{O}_{2}\right)$ $(\mathbf{a}, \mathbf{b})$ in roots and lipid peroxidation (MDA) (c) in four-days old T. aestivum L. (wheat) seedlings of drought tolerant (DT) cv. Ekada 70 and drought sensitive (DS) cv. Salavat Yulaev under normal and drought (12\% PEG-6000) conditions. MDA was estimated in wheat seedlings exposed to drought for $7 \mathrm{~h}$ (c). The bars indicate the mean values of three repetitions \pm SEM. Different lowercase letters on top of the columns indicate that means for each treatment at different time points are different at $p<0.05$. Different capital letters on top of the columns indicate that means for the same time point of various treatments are different at $p<0.05$.

It was found that the plants of DS cv. Salavat Yulaev were more susceptible to the effects of drought, as evidenced by a sharp two-fold drop in AsA throughout the entire experiment and an almost three-fold decrease in GSH (Figure 2d), which correlates with the same level of GSSG (Figure 2b). Evaluation of the AsA and GSH contents under drought conditions in plants of cv. Ekada70 demonstrated drought tolerance-the decrease in the AsA content was about $20 \%$ relative to the control level (Figure $2 \mathrm{a}$ ). While the glutathione system reacts to stress brighter, as evidenced by an average 1.5 times dropping in GSH during the total duration of stress (Figure 2c), and by 2 times increasing of GSSG by $1 \mathrm{~h}$ of stress exposure and a noticeable decrease by $5 \mathrm{~h}$ of stress exposure (Figure 2e). 

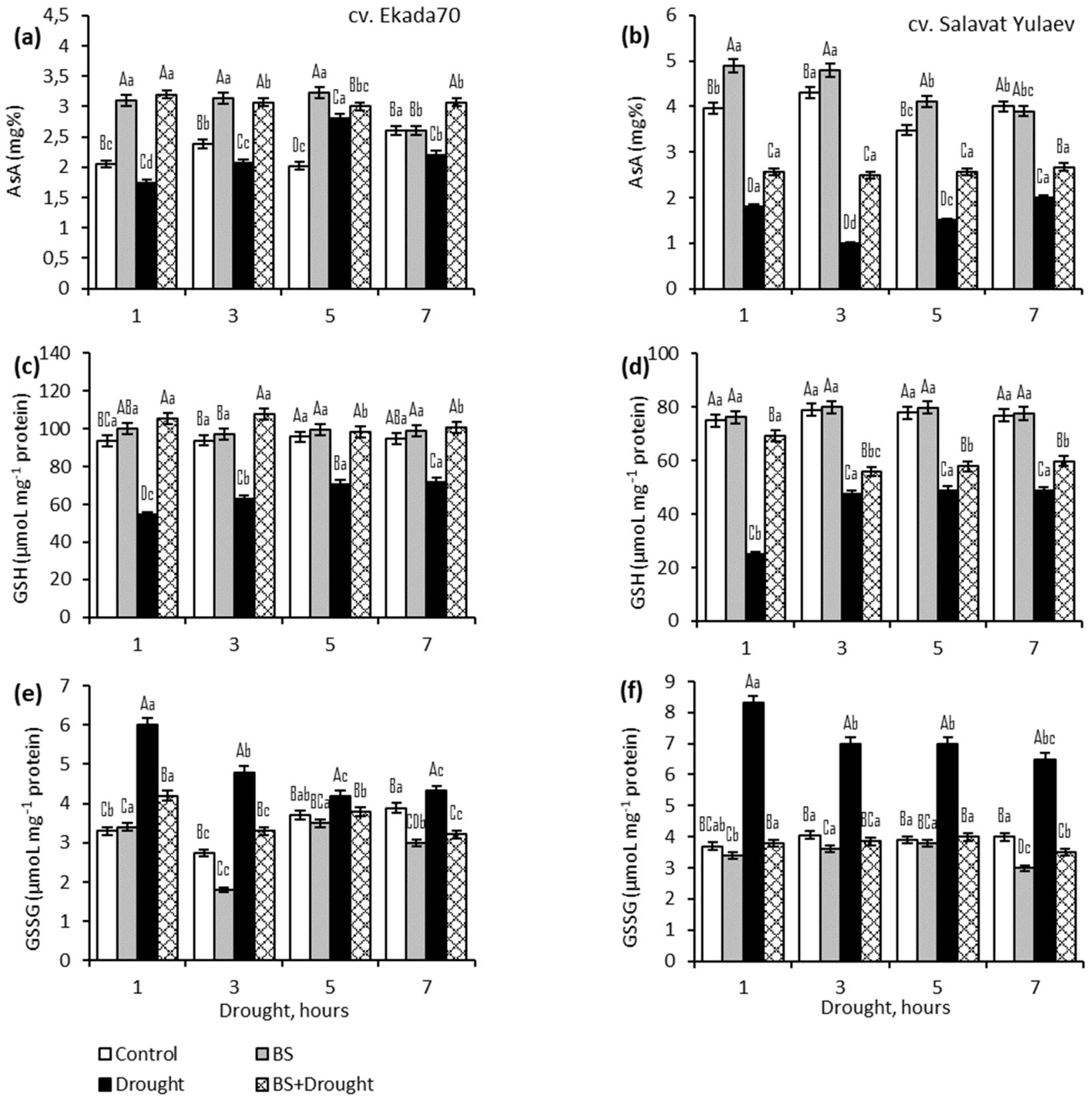

Figure 2. Effect of B. subtilis 10-4 (BS) pre-treatment on the content of some non-enzymatic antioxidants (ascorbate-AsA $(\mathbf{a}, \mathbf{b})$, reduced glutathione-GSH (c,d), and oxidized glutathione-GSSG $(\mathbf{e}, \mathbf{f}))$ in two Triticum aestivum L. early seedlings (four-days old) differing in drought sensitivity (drought tolerant (DT) cv. Ekada70 (a,c,e); drought sensitive (DS) cv. Salavat Yulaev $(\mathbf{b}, \mathbf{d}, \mathbf{f})$ ) grown under normal (control) and drought conditions. The bars indicate the mean values of three repetitions \pm SEM. Different lowercase letters on top of the columns indicate that means for each treatment at different time points are different at $p<0.05$. Different capital letters on top of the columns indicate that means for the same time point of various treatments are different at $p<0.05$.

Under drought conditions, the plants of DS cv. Salavat Yulaev were found to have a sharp decrease of AsA (by 200\%) (Figure 2b) and GSH (by 300\%) (Figure 2d) throughout the entire experiment. In plants of DT cv. Ekada70, drought led to a smaller decrease of AsA (by 20\%) (Figure 2a) and GSH (by 150\%) (Figure 2c) throughout the entire period of stress in comparison with DS genotype. Moreover, it was found that drought exposure for $1 \mathrm{~h}$ increased GSSG in both DT (by 176\%) (Figure 2e) and DS (by 224\%) (Figure 2f) wheat seedlings. However, during further drought exposure (from 3 to $7 \mathrm{~h}$ ) GSSG were varied between $110-170 \%$ and $163-174 \%$ for DT and DS genotypes, respectively. 
B. subtilis 10-4 did not prevent the stress-mediated decrease of AsA in plants of DS cv. Salavat Yulaev (Figure 2b), but rather contributed to the maintenance of its increased level (by $130-250 \%$ ) in comparison with control (non-bacterized) and stressed plants. At the same time, in these plants GSH was higher than the values of stressed plants (Figure 2d), but lower than the control. In parallel, a restoration of GSSG at the level of control values was observed (Figure 2f). In plants of DT cv. Ekada70, pre-treatment with B. subtilis 10-4 stabilized GSH and AsA at the level of control under drought (Figure 2a,c,e).

\subsection{The Activity of Ascorbate Peroxidase (APX) and Glutathione Reductase (GR)}

The results presented in Figure 3 indicate a pronounced activation of APX and GR in both wheat genotypes (Figure 3), but the response amplitude was higher in seedlings of DS cv. Salavat Yulaev (Figure 3b,d), where drought caused a more than $300 \%$ and $200 \%$ increase in APX and GR activities, respectively. In contrast, in seedings of cv. Ekada70 the activities of APX and GR increased approximately by $150 \%$ and $120-170 \%$, respectively (Figure 3c). Furthermore, the reduction of GR activity in both genotypes after $3 \mathrm{~h}$ of stress exposure was observed (Figure $3 \mathrm{c}, \mathrm{d}$ ).
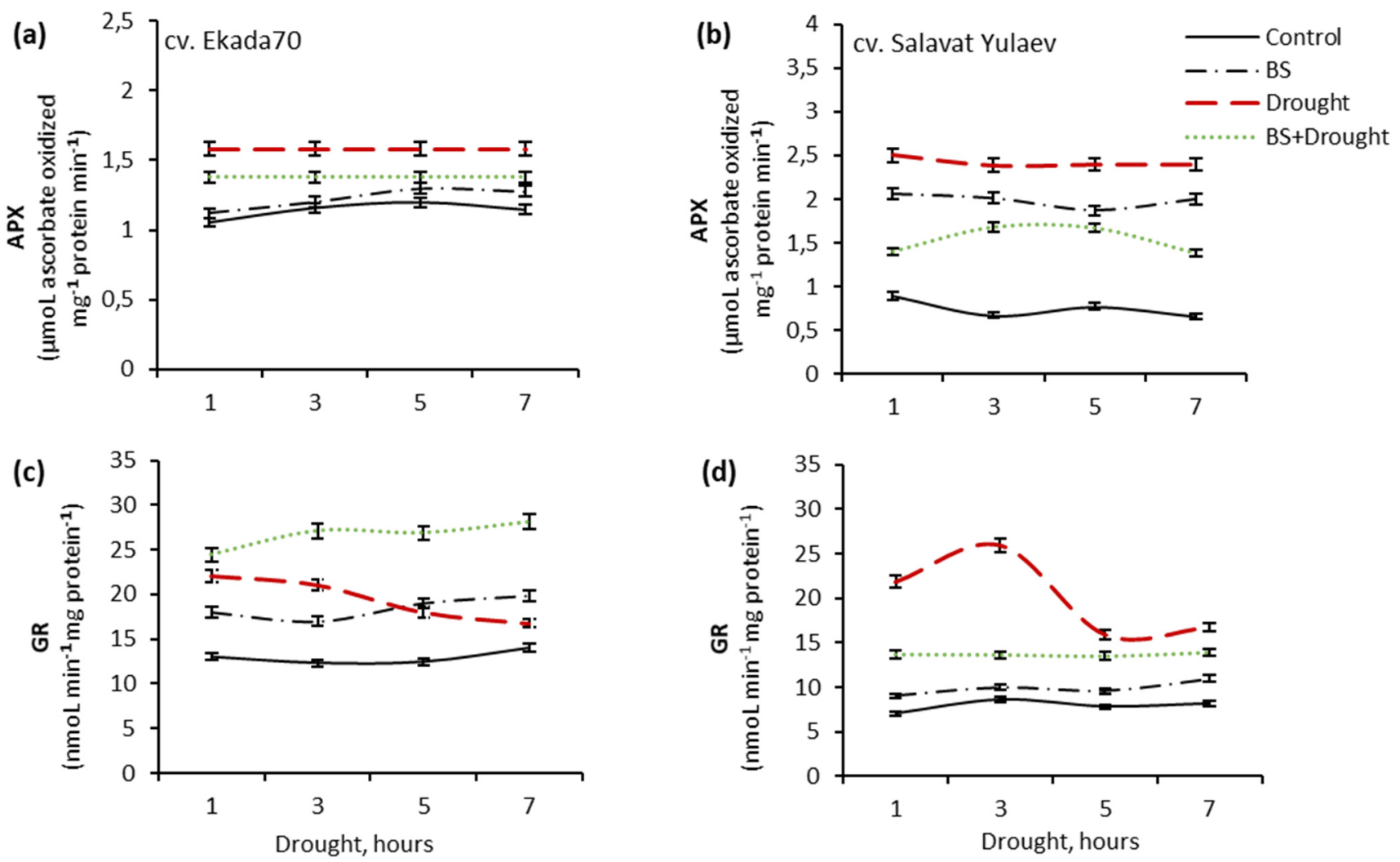

Figure 3. Influence of B. subtilis 10-4 (BS) pre-treatment on the activity of ascorbate peroxidase (APX) (a,b) and glutathione reductase (GR) (c,d) in two T. aestivum L. seedlings (four-days old) differing in drought sensitivity (drought tolerant (DT) cv. Ekada 70 (a,c); drought sensitive (DS) cv. Salavat Yulaev (b,d)) grown under normal (control) and drought (12\% PEG-6000) conditions. The bars indicate the mean values of three repetitions $\pm \mathrm{SEM}$.

Pre-treatment with B. subtilis 10-4 under drought led to a decrease in APX (by 142$173 \%$ ) and GR (by 120-192\%) activities in plants of DS cv. Salavat Yulaev (Figure 3b,d). Whereas, in plants of DT cv. Ekada70, a smaller decrease (by 115-120\% under control) in APX activity and an additional activation of GR (by 125-130\%) were observed (Figure 3a,c). Bacterial treatment significantly reduced (by 114-140\%) stress-caused APX activity in seedlings of cv. Ekada70 (Figure 3a) and resulted in additional GR activation in them, reaching a $200 \%$ increase by $7 \mathrm{~h}$ of stress exposure (Figure 3c). It should be noted, under normal growth conditions B. subtilis 10-4 resulted in different activations of APX and GR 
in both wheat genotypes: by $260 \%$ and $140 \%$, respectively, in plants of cv. Salavat Yulaev (Figure $3 \mathrm{~b}, \mathrm{~d}$ ); by $115 \%$ and $150 \%$, respectively, in plants of cv. Ekada70 (Figure $3 a, c)$.

\section{Discussion}

Drought is a major environmental stress that limits plant growth and inhibits many other cellular developmental functions with the effect of reducing crop productivity worldwide [1-5]. In our work, we used two wheat genotypes, which differ significantly in the degree of drought sensitivity (drought-tolerant (DT) cv. Ekada70 and drought-susceptible (DS) cv. Salavat Yulaev)). It thus comes as no surprise that drought stress led to significant inhibition of seedling growth in both cultivars. The application of B. subtilis 10-4 had a growth-stimulating effect under normal conditions and protective effect under drought conditions on both DS and DT early wheat seedlings. These results are consistent with other studies in which a marked increase in wheat plant growth and tolerance was observed in response to PGPB treatment $[10,16]$. However, our results also suggest wheat genotype (cultivar)-specific physiological action of the same strain B. subtilis 10-4 on seed germination showing the best positive effect on DT cv. Ekada70.

It is of great interest to compare two wheat genotypes contrasting in drought sensitivity according to the state of the components of the antioxidant system, as this system is one of the most critical mechanisms that confer resistance/tolerance to stress in plants. The intensity of oxidative processes in the cell can be judged by the content of the end product of lipid peroxidation-MDA, as well as hydrogen peroxide $\left(\mathrm{H}_{2} \mathrm{O}_{2}\right)$ and superoxide $\left(\mathrm{O}_{2}{ }^{\bullet-}\right)$, which are normally present in tissues in rather low concentrations. In many studies in stressed plants, the contents of MDA and $\mathrm{H}_{2} \mathrm{O}_{2}$ notably declined thanks to PGPB treatment $[10,14,17-19]$. For example, non-bacterized and drought exposed okra plants generated ROS to a more lethal level than in bacterized plants [19]. The findings indicate that PGPB alters the rate of metabolism by activating a strong ROS scavenging system in stressed plants. These may accelerate the restoration of membrane stability and protect against photodamage during re-watering in bacterial treated plants [19]. Our results also showed $B$. subtilis decreases lipid peroxidation and $\mathrm{H}_{2} \mathrm{O}_{2}$ in wheat of both genotypes under drought (Figure 1). Interestingly, the bacterial introduction (before stress) was able to independently activate some antioxidant enzymes. The ability of $B$. subtilis under normal growth conditions to slightly increase $\mathrm{H}_{2} \mathrm{O}_{2}$ in roots of wheat seedlings (Figure 1) may play an important role in biochemical processes associated with biosynthesis of suberin and lignin [20]. This can help to strengthen the cell walls and subsequently ensure effective neutralization of the excess (damaging) stress-induced ROS increase, as well as prevent disruption of the integrity of cells' membrane structures and changes in their permeability under stress conditions. In favor of this, in our previous study, we demonstrated B. subtilis 10-4 intensified lignin deposition in roots of bean plants under normal conditions and exerted a protective effect under salinity decreasing oxidative damages [21]. Perhaps $B$. subtilis is perceived by plants as an agent that causes initial intracellular changes in the antioxidant system which, however, do not lead to seedling damage (Figure 1). Thus, a low ROS production may be important for the pre-adaptive action of Bacillus on plants to subsequent stressful situations since ROS are known to act as signaling molecules in the initiation of a cascade of defense reactions in plants [5,6]. However, a sharp stressinduced increase in ROS can lead to serious destructive consequences $[1,5,6]$. There is now substantial evidence that different plants inoculated with various PGPBs can survive under abiotic stress-caused oxidative damages by acting on antioxidant enzymes $[10,19,22,23]$. The increased activity of SOD, POD, CAT, APX, and GR in PGPB-inoculated plants has been reported as one of the main mechanisms of microbe-induced plant protection against drought $[7,10,18,22]$.

The key role in stabilizing the redox status is assigned to non-enzymatic antioxidants AsA and GSH, which are involved in the direct neutralization of an excess of ROS, and are also the most important participants in the AsA-GSH cycle (or Halliwell-Assad pathway) $[5,6]$. In this cycle, APX catalyzes the reduction of $\mathrm{H}_{2} \mathrm{O}_{2}$ into the water with 
reduced AsA acting as an electron donor. Dehydroascorbate reductase (DHAR) utilizes the electrons provided by GSH to reduce dehydroascorbic acid (DHA), which is previously produced from monodehydroascorbate (MDHA). Simultaneously, reduced GSH is oxidized into GSSG by DHAR, and GSSG is then reduced into GSH, catalyzed by GR. The AsA-GSH cycle can maintain an appropriate oxidative and reductive environment in cells through regulating three interdependent redox couples: AsA/DHA, GSH/GSSG, and $\mathrm{NAD}(\mathrm{P}) \mathrm{H} / \mathrm{NAD}+(\mathrm{P})[6]$. According to the literature data, the accumulation of AsA and GSH in drought-stressed okra plants inoculated with $B$. subtilis indicates activation of the AsA-GSH cycle, promoting active trapping of drought-generated $\mathrm{H}_{2} \mathrm{O}_{2}$ [19]. $B$. amyloliquefaciens 5113 had a protective effect on wheat plants during drought by regulating the activity of the ascorbate-peroxidase APX1 gene and enzymes of the AsA-GSH complex due to the high intracellular content of AsA and GSH, which provides a high buffer redox capacity of cells and serves as a key player in the antioxidant defense system [24]. Interestingly, under normal conditions, B. velezensis 5113 had practically no effect on the AsA-GSH cycle in wheat but significantly reduced APX activity under heat stress. However, under drought and cold stress conditions, there was no significant decrease was found [25]. In addition, it was reported that drought led to an increase in the activity of such stress-related genes as APX1, SAMS1, and HSP17.8 in wheat leaves and increased the activity of the AsA-GSH cycle' enzymes. While in the same bacterial-treated plants, the number of transcripts of these genes decreased, which indicates an improvement in the state of homeostatic mechanisms due to bacterial priming [26]. Obviously, Bacillus strains can reduce the degree of oxidative damage in different ways with the involvement of various biochemical pathways, which may depend on many factors, including the characteristics of the strain itself, the type of plant, the type of stress, and its intensity. Our results revealed that DT cv. Ekada70 has more GSH and increased GR activity, and this possibly determines a slight drop in AsA and GSH under stress (Figure 2). Most likely this determines drought tolerance of cv. Ekada70 in comparison with cv. Salavat Yulaev, which is declared drought-susceptible. This is also supported by obtained data on the pronounced drop in AsA and GSH levels in drought-stressed cv. Salavat Yulaev. Drought led to the strongest APX and GR activation, but the response amplitude was higher in DS wheat plants than in DT ones. The result of these enzymes' work was reflected in $\mathrm{H}_{2} \mathrm{O}_{2}$, MDA, AsA, and GSH, as well as the growth rates of these plants. Assessment of AsA and GSH in bacterized plants under drought showed that their level was higher compared to non-bacterized plants under the same conditions. In plants of the DS cv. Salavat Yulaev, $B$. subtilis promotes an increase in the level of antioxidants and practically restores the level of GSSG. We associate this effect with the fact that B. subtilis 10-4 significantly reduced the activity of APX and GR, and these antioxidants were depleted to a much lesser extent. It should be noted that in these plants, under normal growth conditions, B. subtilis 10-4 significantly increased APX activity, while GR was slightly activated. In seedlings of cv. Ekada70, the bacterial treatment led to an almost complete restoration of the AsA and GSH contents. We suggest this effect with the fact that bacteria under normal growth conditions led to a small but reliable additional AsA accumulation and its activation by GR, which, in our opinion, provides rapid complete recovery of GSH and decrease in GSSG levels. Thus, the results indicate the key role of AsA and GSH in the formation of B. subtilis-induced drought tolerance of wheat genotypes differing in drought sensitivity.

\section{Materials and Methods}

\subsection{Bacterial Strain and Inoculum Preparation}

The endophytic bacterium B. subtilis (strain 10-4) was isolated from the arable soils at the Bashkir Research Institute of Agriculture of the Ufa Federal Research Center at the Russian Academy of Sciences (BRIA UFRC RAS) (Ufa, Russia), identified using $16 \mathrm{~S}$ rRNA, characterized [14,18] and deposited in the Russian National Collection of Industrial Microorganisms (VKPM) (registration number B-12988). B. subtilis 10-4 cells were cultured in LB solid agar medium at $37^{\circ} \mathrm{C}$ for $24 \mathrm{~h}$ [27]. To obtain inoculum of B. subtilis 10-4, the 
suspension was prepared containing $10^{8} \mathrm{CFU} \mathrm{mL}{ }^{-1}$ of bacteria according to $0.5 \mathrm{McF}$ arland turbidity standard and monitored at the optical density $600 \mathrm{~nm}$ (OD600) (SmartSpecTM Plus spectrophotometer, Bio-Rad, USA), and then diluted down to $10^{5} \mathrm{CFU} \mathrm{mL}{ }^{-1}$ using sterile water.

\subsection{Plant Materials and Growth Conditions}

Wheat seeds (Triticum aestivum L., BBAADD $2 n=42$ ) of drought-susceptible (DS) cv. Salavat Yulaev (SY,) and drought-tolerant (DT) cv. Ekada70 were obtained from the Chishminsky Breeding Station, UFRC RAS (Russia). The seeds were sterilized in 97\% ethyl alcohol for $1 \mathrm{~min}$, then washed with sterile tap water for 2-3 min (until the smell of alcohol disappeared). Thereafter, the seeds were immersed into solutions of $B$. subtilis 10-4 $\left(10^{5} \mathrm{CFU} \mathrm{mL}{ }^{-1}\right)$ or sterile water (control) for $1 \mathrm{~h}$. The treated seeds were hydroponically grown (on filter paper moistened with tap water) under a 16/8 h light/dark photoperiod regime under $\left(200 \mu \mathrm{moL} \mathrm{m}^{-2} \mathrm{~s}^{-1}\right)$ at $22-24{ }^{\circ} \mathrm{C}$ for four days. Thereafter, the seedlings were transferred to glasses with distilled water (control) or 12\% PEG-6000 (drought) and grew further at the same conditions. Plant samples (root, shoots, or whole seedlings) were taken after $0.5,1,2,3,5,7 \mathrm{~h}$ (depending on purposes) to assess physio-biochemical attributes.

\subsection{Growth Parameters}

To assess wheat seed germination percentage, the seeds pre-treated with B. subtilis 10-4 and non-treated (control) germinated in Petri dishes in the presence of 12\% PEG-6000 (drought) and distilled water (normal growth condition) for three days. The length of wheat seedlings' roots and shoots were measured with a ruler in four-days old hydroponically grown seedlings exposed to drought for $7 \mathrm{~h}$. Fresh weight (FW) of roots and shoots were recorded on the same day. To measure the dry weight (DW), samples were placed in an oven at $70{ }^{\circ} \mathrm{C}$, and after $48 \mathrm{~h}$ measurements were conducted [28].

\subsection{Malondialdehyde (MDA) and Hydrogen Peroxide $\left(\mathrm{H}_{2} \mathrm{O}_{2}\right)$ Contents}

MDA and $\mathrm{H}_{2} \mathrm{O}_{2}$ were measured as biomarkers of lipid peroxidation and oxidative stress. To determine MDA [28], wheat seedlings were ground in distilled water and then homogenized in $20 \%$ trichloroacetic acid. The homogeneous samples then were centrifuged $(10,000 \times g, 10 \mathrm{~min})$. Then the supernatant was mixed with $0.5 \%$ thiobarbituric acid prepared in $20 \%$ trichloroacetic acid and was kept in a boiling water bath $\left(100{ }^{\circ} \mathrm{C}\right.$, $30 \mathrm{~min}$ ), and quickly cooled. Absorbance was spectrophotometrically (SmartSpecTM Plus, Bio-Rad, CA, USA) measured at $532 \mathrm{~nm}$ and $600 \mathrm{~nm}$. The MDA was expressed as nmoL g ${ }^{-1}$ FW.

To determine $\mathrm{H}_{2} \mathrm{O}_{2}$ [29], samples of plant material (roots) homogenized (1:5 weight/volume) in $0.05 \mathrm{M}$ sodium phosphate buffer (FB), $\mathrm{pH}$ 6.2. The supernatant was separated by centrifugation (Eppendorf ${ }^{\circledR}$ Microcentrifuge $5415 \mathrm{R}$, Humburg, Germany) at 15,000 $\times \mathrm{g}$ for $15 \mathrm{~min}$. The concentration of $\mathrm{H}_{2} \mathrm{O}_{2}$ in the supernatant was spectrophotometrically (SmartSpecTM Plus, Bio-Rad, Hercules, CA, USA) determine using xylenol orange in the presence of $\mathrm{Fe}^{2+}$ at $560 \mathrm{~nm}$. The $\mathrm{H}_{2} \mathrm{O}_{2}$ was expressed as $\mu \mathrm{moL} \mathrm{g}^{-1} \mathrm{FW}$.

\subsection{Measurement of Non-Enzymatic Antioxidants \\ 4.5.1. Total of Reduced Glutathione (GSH) and Oxidized Glutathione (GSSG) Content}

Content of GSH and GSSG forms of glutathione from the same plant sample were determined using a spectrofluorimetric method based on the formation of a fluorescent product o-phthalaldehyde (Sigma, Australia) dependent on $\mathrm{pH}$ of the medium. Plant samples $(0.5 \mathrm{~g})$ were homogenized in $4 \mathrm{~mL}$ of the mixture containing $0.1 \mathrm{M}$ potassium phosphate buffer ( $\mathrm{pH} 8.0$ ) and 25\% metaphosphoric acid at a ratio of 3.75:1 (by volume) according to Hissin and Hilf [30]. Homogenate was centrifuged for $10 \mathrm{~min}$ at $8000 \times g$, and then supernatant was repeatedly centrifuged for $5 \mathrm{~min}$ at $13,000 \times$ g. GSH and GSSG in the obtained supernatant were quantified using reagents described in detail by Maslennikova et al. [15]. In order to determine the content of GSH and GSSG, we monitored the kinetics 
of the fluorescence strength of the formed complexes at a temperature of $25^{\circ} \mathrm{C}$ using an EnSpire Model 2300 Multilabel Microplate Reader (PerkinElmer, Boston, MA, USA) at $420 \mathrm{~nm}$ (excitation wavelength of $350 \mathrm{~nm}$ ). The content of GSH and GSSG was expressed

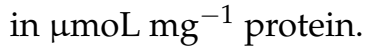

\subsubsection{Ascorbate (AsA) content}

AsA content was determined using the titration method [31]. Plant samples (10 g) were ground in a porcelain mortar, extracted with $10 \mathrm{~mL}$ of distilled water, stirred, and filtered through a paper filter. An amount of $20 \mathrm{~mL}$ of filtrate was taken into a conical flask and $1 \mathrm{~mL}$ of $2 \% \mathrm{HCl}, 0.5 \mathrm{~mL}$ of $1 \% \mathrm{KI}$, and $2 \mathrm{~mL}$ of $0.5 \%$ starch were added, stirred, and titrated with $0.001 \mathrm{moL} \mathrm{L}^{-1} \mathrm{KIO}_{3}$ until stable blue color staining. The ascorbate content was expressed in $\mathrm{mg} \% \mathrm{FW}$.

\subsection{Measurement of Enzymatic Antioxidants}

\subsubsection{Ascorbate Peroxidase (APX) Activity}

Ascorbate peroxidase (APX, EC 1.11.1.11) activity was estimated by monitoring ascorbate oxidation at $290 \mathrm{~nm}$ [32]. The reaction mixture $(2.93 \mathrm{~mL})$ consisted of $50 \mathrm{mM}$ phosphate buffer ( $\mathrm{pH} 7.0),(0.03 \mathrm{~mL}) 17 \mathrm{mM}$ ASC, $(0.03 \mathrm{~mL})$ EDTA, $0.01 \mathrm{~mL}$ extract. The reaction was started by the addition of $0.03 \mathrm{~mL} 0.06 \% \mathrm{H}_{2} \mathrm{O}_{2}$ and was determined during the first $100 \mathrm{~s}$. The results were expressed as $\mu \mathrm{moL}$ ascorbate oxidized $\mathrm{mg}^{-1}$ protein $\mathrm{min}^{-1}$.

\subsubsection{Glutathione Reductase (GR) Activity}

GR (EC 1.6.4.2) activity was determined by following the oxidation of nicotinamide adenine dinucleotide phosphate (NADPH) at $340 \mathrm{~nm}$ (extinction coefficient $6.2 \mathrm{mM} \mathrm{cm}^{-1}$ ) as described by Rao [33]. The $1 \mathrm{~mL}$ assay mixture contained $100 \mathrm{mM}$ potassium phosphate buffer (pH 7.8), $2 \mathrm{mM}$ EDTA, $0.2 \mathrm{mM}$ NADPH, and the plant extract. The assays were initiated by the addition of $0.5 \mathrm{mM}$ GSSG at $25^{\circ} \mathrm{C}$. The activity was measured after monitoring NADPH oxidation for three absorbances was taken at $340 \mathrm{~nm}$ activity expressed as nmoL min $^{-1} \mathrm{mg}_{\text {protein }}{ }^{-1}$.

Activities of all studied antioxidative enzymes were converted to $\mathrm{mg}$ of protein. Total soluble protein was estimated according to the Bradford method [34] by using bovine serum albumin (BSA) as a standard. For spectrophotometric analyses, a spectrophotometer UNICO 2800 (United Products @ Instruments, Middlesex, NJ, USA) was used.

\subsection{Statistical Analysis}

All physiological and biochemical experiments were performed at three biological and three analytical replicates. The data were presented as the mean \pm standard error (SEM). Statistically significant differences between the mean values were evaluated using analysis of variance (ANOVA), followed by the Tukey test $(p<0.05)$.

\section{Conclusions}

Thus, a genotypic specificity of the responses of wheat genotypes contrasting in drought sensitivity to endobacteria $B$. subtilis inoculation under drought was revealed. Which is associated with the ability of B. subtilis to regulate the components of the AsAGSH complex as an effective antioxidant system. The result suggests the key role in the realization of B. subtilis-caused drought tolerance in plants of DS cv. Salavat Yulaev and DT cv. Ekada70 are AsA and GSH, respectively; which in both cases resulted in reduced oxidative damages of membranes and improved wheat plants' growth under drought. The obtained results would be useful in developing drought-tolerant wheat cultivars associated with endobacteria B. subtilis in future breeding programs.

Author Contributions: Conceptualization, methodology, formal analysis, investigation, resources, D.M., and O.L.; writing—original draft preparation, D.M.; writing-review and editing, O.L. All authors have read and agreed to the published version of the manuscript. 
Funding: This research was partially funded by RFBR, grant number 19-016-00035.

Institutional Review Board Statement: Not applicable.

Informed Consent Statement: Not applicable.

Data Availability Statement: Not applicable.

Acknowledgments: The research was carried out within the framework of the state assignment of Russia (registration number AAAA-A21-121011990120-7), using the instrument park of the RCCU "Agidel" and "KODINK" UFRC RAS. We thank Massimo Bosacchi for the English language editing.

Conflicts of Interest: The authors declare no conflict of interest. The funder had no role in the design of the study; in the collection, analyses, or interpretation of data; in the writing of the manuscript, or in the decision to publish the results.

$\begin{array}{ll}\text { Abbreviations } \\ \text { APX } & \text { ascorbate peroxidase } \\ \text { AsA } & \text { ascorbate } \\ \text { DS } & \text { drought susceptible } \\ \text { DT } & \text { drought tolerant } \\ \text { GR } & \text { glutathione reductase } \\ \text { GSH } & \text { glutathione } \\ \text { GSSG } & \text { glutathione disulfide (oxidized glutathione) } \\ \mathrm{H}_{2} \mathrm{O}_{2} & \text { hydrogen peroxide } \\ \text { MDA } & \text { malondialdehyde } \\ \text { PEG } & \text { polyethylene glycol } \\ \text { PGPB } & \text { plant growth-promoting bacteria } \\ \text { ROS } & \text { reactive oxygen species }\end{array}$

\section{References}

1. Farooq, M.; Wahid, A.; Kobayashi, N.; Fujita, D.; Basra, S.M.A. Plant Drought Stress: Effects, Mechanisms and Management. Agron. Sustain. Dev. 2009, 29, 185-212. [CrossRef]

2. FAO. The State of Food and Agriculture. Climate Change, Agriculture and Food Security; FAO: Rome, Italy, 2016; Available online: http:/ / www.fao.org/3/a-i6030e.pdf (accessed on 21 October 2021).

3. Asseng, S.; Martre, P.; Maiorano, A.; Rötter, R.P.; O’Leary, G.J.; Fitzgerald, G.J.; Girousse, C.; Motzo, R.; Giunta, F.; Ali Babar, M.; et al. Climate Change Impact and Adaptation for Wheat Protein. Glob. Chang. Biol. 2019, 25, 155-173. [CrossRef]

4. FAO. Cereal Supply and Demand Brief. 2021. Available online: http://www.fao.org/worldfoodsituation/csdb/ru/ (accessed on 21 October 2021).

5. Hasanuzzaman, M.; Nahar, K.; Gill, S.S.; Gill, R.; Fujita, M. Drought Stress Responses in Plants, Oxidative Stress, and Antioxidant Defense. In Climate Change and Plant Abiotic Stress Tolerance; Tuteja, N., Gill, S.S., Eds.; Wiley-VCH Verlag GmbH \& Co.: Weinheim, Germany, 2014; pp. 209-249.

6. Hasanuzzaman, M.; Bhuyan, M.H.M.B.; Zulfiqar, F.; Raza, A.; Mohsin, S.M.; Mahmud, J.A.; Fujita, M.; Fotopoulos, V. Reactive Oxygen Species and Antioxidant Defense in Plants under Abiotic Stress: Revisiting the Crucial Role of a Universal Defense Regulator. Antioxidants 2020, 9, 681. [CrossRef]

7. Lastochkina, O.; Aliniaeifard, S.; Kalhor, M.S.; Yuldashev, R.; Pusenkova, L.; Garipova, S. Plant Growth Promoting Bacteria Biotic Strategy to Cope with Abiotic Stresses in Wheat. In Wheat Production in Changing Environments: Management, Adaptation and Tolerance; Hasanuzzaman, M., Nahar, K., Hossain, A., Eds.; Springer: Singapore, 2019; pp. 579-614.

8. Sood, G.; Kaushal, R.; Sharma, M. Significance of Inoculation with Bacillus subtilis to Alleviate Drought Stress in Wheat (Triticum aestivum L.). Vegetos 2020, 33, 782-792. [CrossRef]

9. Goswami, M.; Deka, S. Plant Growth-Promoting Rhizobacteria-Alleviators of Abiotic Stresses in Soil: A Review. Pedosphere 2020, 30, 40-61. [CrossRef]

10. Rashid, U.; Yasmin, H.; Hassan, M.N.; Naz, R.; Nosheen, A.; Sajjad, M.; Ilyas, N.; Keyani, R.; Jabeen, Z.; Mumtaz, S.; et al. Drought-tolerant Bacillus megaterium Isolated from Semi-Arid Conditions Induces Systemic Tolerance of Wheat Under Drought Conditions. Plant Cell Rep. 2021, 1-21. [CrossRef]

11. Bokhari, A.; Essack, M.; Lafi, F.F.; Andres-Barrao, C.; Jalal, R.; Alamoudi, S.; Razali, R.; Alzubaidy, H.; Shah, K.H.; Siddique, S.; et al. Bioprospecting Desert Plant Bacillus Endophytic Strains for Their Potential to Enhance Plant Stress Tolerance. Sci. Rep. 2019, 9, 18154. [CrossRef]

12. Bukhat, S.; Imran, A.; Javaid, S.; Shahid, M.; Majeed, A.; Naqqash, T. Communication of Plants with Microbial World: Exploring the Regulatory Networks for PGPR Mediated Defense Signaling. Microbiol. Res. 2020, 238, 126486. [CrossRef] 
13. Blake, C.; Christensen, M.N.; Kovács, Á. Molecular Aspects of Plant Growth Promotion and Protection by Bacillus subtilis. Mol. Plant-Microbe Interact. 2021, 34, 15-25. [CrossRef]

14. Lastochkina, O.; Garshina, D.; Ivanov, S.; Yuldashev, R.; Khafizova, R.; Allagulova, C.; Fedorova, K.; Avalbaev, A.; Maslennikova, D.; Bosacchi, M. Seed Priming with Endophytic Bacillus subtilis Modulates Physiological Responses of Two Different Triticum aestivum L. Cultivars Under Drought Stress. Plants 2020, 9, 1810. [CrossRef]

15. Maslennikova, D.R.; Plotnikov, A.A.; Shakirova, F.M. Comparative Analysis of the Physiological Effect of Nitric Oxide and 6-Benzylaminopurine on the Components of the Glutathione Complex in the Roots of Wheat Seedlings. Agrokhimiya $2019,3,37$.

16. Li, Y.; Shi, H.; Zhang, H.; Chen, S. Amelioration of Drought Effects in Wheat and Cucumber by the Combined Application of Super Absorbent Polymer and Potential Biofertilizer. PeerJ 2019, 7, e6073. [CrossRef] [PubMed]

17. Shukla, N.; Awasthi, R.P.; Rawat, L.; Kumar, J. Biochemical and physiological responses of rice (Oryza sativa L.) as influenced by Trichoderma harzianum under drought stress. Plant Physiol. Biochem. 2012, 54, 78-88. [CrossRef]

18. Lastochkina, O.; Pusenkova, L.; Yuldashev, R.; Babaev, M.; Garipova, S.; Blagova, D.; Khairullin, R.; Aliniaeifard, S. Effects of Bacillus subtilis on Some Physiological and Biochemical Parameters of Triticum aestivum L. (Wheat) Under Salinity. Plant Physiol. Biochem. 2017, 121, 80-88. [CrossRef] [PubMed]

19. Puthiyottil, P.; Akkara, Y. Pre treatment with Bacillus subtilis mitigates drought induced photo-oxidative damages in okra by modulating antioxidant system and photochemical activity. Physiol. Mol. Biol. Plants 2021, 27, 945-957. [CrossRef]

20. Cabane, M.; Afif, D.; Hawkins, S. Lignins and abiotic stresses. In Lignins Biosynthesis, Biodegradation and Bioengineering; Jouanin, L., Lapierre, C., Eds.; Academic Press, Advances in Botanical Research: London, UK, 2012; Volume 61, pp. $219-262$.

21. Lastochkina, O.; Aliniaeifard, S.; Garshina, D.; Garipova, S.; Pusenkova, L.; Allagulova, C.; Fedorova, K.; Baymiev, A.; Koryakov, I.; Sobhani, M. Seed priming with endophytic Bacillus subtilis strain-specifically improves growth of Phaseolus vulgaris plants under normal and salinity conditions and exerts anti-stress effect through induced lignin deposition in roots and decreased oxidative and osmotic damages. J. Plant Physiol. 2021, 263, 153462. [CrossRef]

22. Kaushal, M. Portraying Rhizobacterial Mechanisms in Drought Tolerance: A Way Forward Toward Sustainable Agriculture. In PGPR Amelioration in Sustainable Agriculture; Singh, A.K., Rumar, A., Singh, P.K., Eds.; Elsevier Inc.: Amsterdam, The Netherlands, 2019; pp. 195-216. [CrossRef]

23. Kim, K.; Jang, Y.-J.; Lee, S.-M.; Oh, B.-T.; Chae, J.-C.; Lee, K.-J. Alleviation of Salt Stress by Enterobacter sp. EJ01 in Tomato and Arabidopsis is Accompanied by Up-Regulation of Conserved Salinity Responsive Factors in Plants. Mol. Cell 2014, 37, 109-117. [CrossRef] [PubMed]

24. Zhou, C.; Ma, Z.; Zhu, L.; Xia, X.; Xie, Y.; Zhu, J.; Wang, J. Rhizobacterial Strain Bacillus megaterium BOFC15 Induces Cellular Polyamine Changes That Improve Plant Growth and Drought Resistance. Int. J. Mol. Sci. 2016, 17, 976. [CrossRef]

25. Abd El-Daim, I.A.; Bejai, S.; Meijer, J. Bacillus velezensis 5113 Induced Metabolic and Molecular Reprogramming During Abiotic Stress Tolerance in Wheat. Sci. Rep. 2019, 9, 16282. [CrossRef] [PubMed]

26. Kasim, W.A.; Osman, M.E.; Omar, M.N.; Abd El-Daim, I.A.; Bejai, S.; Meijer, J. Control of Drought Stress in Wheat Using Plant-Growth-Promoting Bacteria. J. Plant Growth Regul. 2013, 32, 122-130. [CrossRef]

27. Netrusov, A.I.; Egorova, M.A.; Zakharchuk, L.M. Praktikum po Mikrobiologii (A Practical Course in Microbiology); Izdat. Tsentr Akademiya: Moscow, Russia, 2005; 608p.

28. Mokronosova, A.T. Small Workshop on Plant Physiology; Moscow State University: Moscow, Russia, 1994; 184p.

29. Bindschedler, L.V.; Minibaeva, F.; Gardner, S.L.; Gerrish, C.; Davies, D.R.; Bolwell, G.P. Early Signalling Events in the Apoplastic Oxidative Burst in Suspension Cultured French Bean Cells Involve cAMP and Ca ${ }^{2+}$. New Phytol. 2001, 151, 185-194. [CrossRef] [PubMed]

30. Hissin, P.J.; Hilf, R.A. A Fluorometric Method for Determination of Oxidize and Reduced Glutathione in Tissues. Anal. Biochem. 1976, 74, 214-226. [CrossRef]

31. Pleshkov, B.P. Workshop on Plant Biochemistry; Izdat. Colos: Moscow, Russia, 1976; 256p.

32. Verma, S.; Dubey, R.S. Lead Toxicity Induces Lipid Peroxidation and Alters the Activities of Antioxidant Enzymes in Growing Rice Plants. Plant Sci. 2003, 164, 645-655. [CrossRef]

33. Rao, M.V.; Paliyath, G.; Ormrod, D.P. Ultraviolet-B- and Ozone-induced Biochemical Changes in Antioxidant Enzymes of Arabidopsis Thaliana. Plant Physiol. 1996, 110, 125-136. [CrossRef] [PubMed]

34. Bradford, M.M. A Rapid and Sensitive Methods for Quantitation of Microgram Quantities of Protein Utilizing the Principle of Protein Dye Binding. Anal. Biochem. 1976, 74, 248-254. [CrossRef] 